PALABRAS CLAVE I CHAITÉN • URBANISMO SUSTENTABLE - POLITICAS URBANAS • MINISTERIO DE VIVIENDA Y URBANISMO $•$ NUEVAS CIUDADES

KEYWORDS | CHAITÉN • SUSTAINABLE URBANISM • URBAN POLICIES • MINISTRY OF HOUSING AND URBAN PLANNINC CHILE • NEW CITIES
The dilution of the emergency. Urban visions

\section{| RESUMEN |}

El artículo se plantea como un relato crítico al proceso de implementación de una nueva capital para la Provincia de Palena, desde la erupción del volcán Chaitén, que destruyó gran parte de la ciudad del mismo nombre, hasta el descarte del proyecto por las autoridades del Estado en diciembre de 20IO. Esta mirada se hace a título personal, siendo el autor parte del equipo de trabajo del Ministerio de Vivienda y Urbanismo, hasta el año 2010.

\section{| ABSTRACT |}

This article is a critical account of the process of building a new capital city for the Province of Palena, in the Chilean Patagonia. from the eruption of the Chaiten Volcano which destroyed the homonym city, to the decision by state authorities to reject the project in december 2010 . This is a personal view from the author who was part of the team that worked for the chilean Ministry of Housing and Urban Planning.

\title{
La dilución de la emergencia
}

\author{
Artículo de opinión: Visiones urbanas
}

$T_{\mathrm{d}}^{\mathrm{e}}$

erminé el año dos mil diez con un periplo de varios días por la zona andina de La Araucanía. Hicimos junto a mis compañeros de viaje un circuito en dirección a la cordillera; saliendo de la ruta 5 por Victoria, pasando por Curacautín, luego el túnel Las Raíces, la laguna Galletué, araucarias, laguna Icalma, tormentas de granizo, Melipeuco, Cunco y de vuelta a ruta 5 por Freire. Un poco antes de llegar a la Panamericana, entre Melipeuco y Cunco nos detuvimos en un pequeño caserío que nos llamó la atención en un primer vislumbro por sus casas de estilo suizo, pintadas a varios colores. Al observar detenidamente, impresiona su coherente distribución. El pueblo se llama Villa García, y según pude averiguar en la red, fue una de las últimas obras urbanas del Padre Bernabé de Lucerna, quien además de fundar en la región 32 escuelas y dos iglesias, también participó en la fundación de la ciudad de Panguipulli, y colaboró fuertemente en la implementación de equipamientos en Cunco.

El caserío de alrededor de 1.000 habitantes fue diseñado por el religioso capuchino en la década de 1960 con el objetivo preciso de dotar de casas y terrenos para el autosustento de 20 campesinos independizados de sus fundos y organizados por el religioso ${ }^{[1]}$.

Con su arquitectura de estilo suizo, no sólo se les dotó de casas y predios; en el centro del trazado urbano con forma de pista atlética alargada, está la capilla de San Conrado con su característica torre capuchina. Al poniente de ésta una cancha de fútbol y al oriente una plaza que la separa del cruce del camino entre Cunco y Melipeuco; en las esquinas de este cruce, centros educacionales y al otro lado del camino un centro comunitario.

En entrevista a un amigo suyo, éste relataba que el sacerdote citaba como cimiento inspirador de esta iniciativa al «(...) milagro alemán de que tanto se habla (...) se debe en mucha parte a los planes agrarios imaginados y realizados por las grandes instituciones religiosas, católicas y protestantes, con el fin de dar a cada ciudadano una casa y un trozo de terreno. Eso levantó el espíritu del pueblo abatido por las penurias de la Segunda Guerra Mundial. Moral y económicamente, ésa fue la varita mágica que levantó con rapidez la prosperidad alemana» ${ }^{[2]}$.

\footnotetext{
* Arquitecto y Magíster en Arquitectura de la Pontificia Universidad Católica de Chile, 2003. Estudia la relación entre diseño del espacio, el poder y la política. Participó entre junio de 2009 y diciembre de 2010 en el diseño, y gestión del Plan Maestro de la Ciudad de Chaitén el cual coordinó desde el MINVU. Socio fundador de Mapa Consultores, ha proyectado obras arquitectónicas así como en proyectos complejos de paisajes y ciudades. Actualmente es docente de la Escuela de Arquitectura de la Universidad Diego Portales.

[1] http://lbnpanguipulli.cl/index.php?option $=$ com content\&view $=$ article\&id $=293 \&$ catid $=1 \&$ ltemid $=216$

[2] http://lbnpanguipulli.cl/index.php?option $=\mathrm{com}^{-}$content\&view $=$article\&id $=311$ \&catid $=1$ \&ltemid $=216$
} 
1. Padre Bernabé de Lucerna la Orden al Mérito Bernardo O'Higgins que le concedió el Gobierno del Presidente Ibáñez en 1958. Fuente: Publicación periodística sin fecha, posiblemente de las revistas Vea o Ercilla de algún mes de 1965 reproducido por web Las Buenas Noticias de Panguipulli.

2. Padre Bernabé de Lucerna aparentemente recorriendo Villa García. En el pie de foto dice «el padre Bernabé contempla una de sus obras (...)». Fuente: Publicación periodística sin fecha, posiblemente de las revistas Vea o Ercilla de algún mes de 1965 reproducido por web Las Buenas Noticias de Panguipulli.

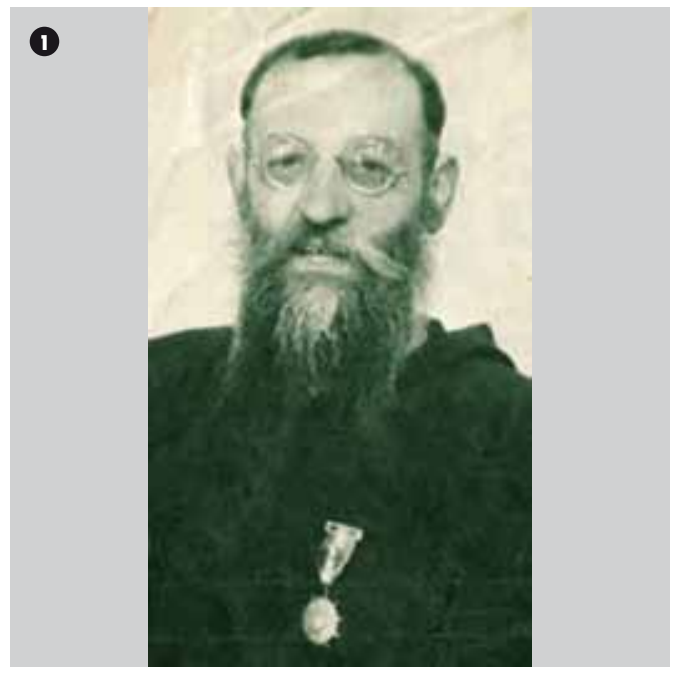

Esta singular iniciativa urbana -a la que hoy aspira la mirada y el actuar urbano sustentable, debido a su escala- plantea una nítida ecuación de objetivos, actores, gestión, y concreción. Todo lo que uno aspiraría hoy cincuenta años después. Misma aspiración que fue la base con la que se actuó en el proceso latente de la implementación de una nueva capital para la Provincia de Palena, la Provincia de los Parques.

El objetivo de este artículo es dar a conocer el proceso de vaivenes del proyecto de Santa Bárbara; que es un caso singular dentro de la historia del país al igual que Villa García, que se pretendía fundar desde cero, pero a diferencia de ésta incluye una complejidad dada por las vicisitudes de la política.

\section{NACIMIENTO DE LA EMERGENCIA}

El volcán Chaitén hizo erupción explosivamente el 2 de mayo de 2008 después de una serie de temblores detectados en Chaitén, Palena y Futaleufú. Descargó cenizas y material incandescente en sucesivas explosiones, y desde su cono se desprendió una pluma de cenizas que llegaba hasta la provincia de Buenos Aires, en Argentina, así como evacuó material por el río Blanco, material que fue el causante del desastre del poblado al taponear el río que ahora salía por toda la trama urbana, llenando sus calles de cenizas y, finalmente, cortando la ciudad en dos.

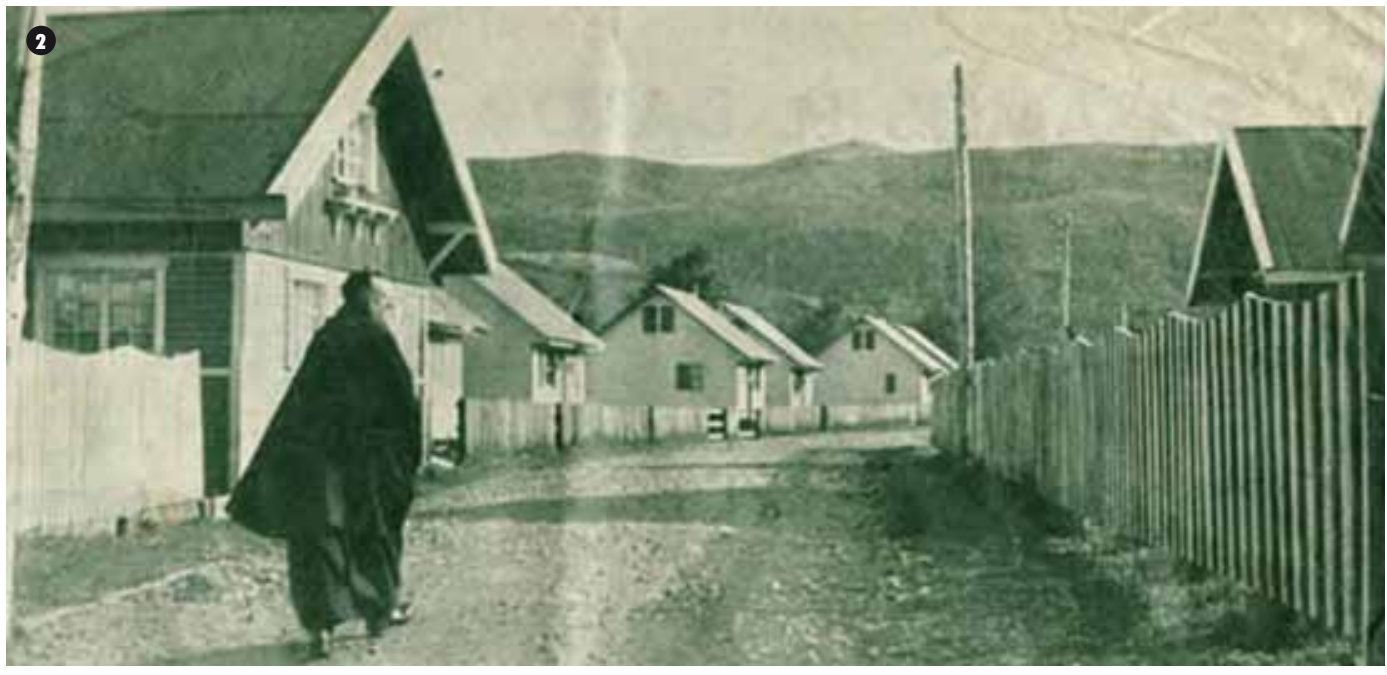

Tras la erupción se decretó la alerta roja que derivó en una operación liderada por un ministro en campaña (José Goñi, Ministro de Defensa de la época) que evacuó la población retirando en dos días cerca de 4.000 personas, que fueron ubicadas en Puerto Montt y Chiloé en una primera instancia.

Posterior a ello se nombró una delegada presidencial, Paula Narváez, que en conjunto con la Intendencia Regional, estuvo encargada de la coordinación de los beneficios estatales para los desplazados así como coordinar las tareas de reconstrucción de la Provincia de Palena. Éstos consistieron en un subsidio habitacional para ser ubicado en cualquier parte del territorio nacional, un bono familiar y finalmente, la implementación de la ley Chaitén aprobada por el Congreso, la cual permitió al Estado la compra de los predios afectados por la erupción a un valor previo a la catástrofe.

En paralelo se dieron los primeros pasos para la planificación del futuro de la provincia. La Pontificia Universidad Católica de Chile y la Universidad Austral, junto a un grupo de especialistas internacionales, trabajaron en un estudio que permitió evaluar escenarios de retorno, para la reconstrucción o relocalización de Chaitén ${ }^{[3]}$.
En el estudio se analizaron cinco alternativas para la nueva capital provincial: Chaitén Norte, Fandango, Santa Bárbara Sur, Santa Bárbara y Bahía Pumalín. Cada una se sometió a un análisis comparativo enfocado en explorar las ventajas y desventajas de las diferentes opciones, en cuanto a: medio ambiente, economía y viabilidad, cultura y sociedad, y recursos naturales.

Se aplicó, para las cinco localizaciones, el Modelo Estratégico para el Análisis de Localizaciones (MEAL), el cual analiza de manera estratégica, el desempeño de cada área en relación a criterios específicos en los ámbitos anteriormente nombrados.

Los criterios seleccionados para el proceso de evaluación se basaron en una mirada integral de sustentabilidad, en relación a la selección de una localización para un futuro desarrollo urbano. La lista final de criterios fue validada con el grupo de representantes de las autoridades que conformaron el comité estratégico.

Dentro de las cinco opciones los resultados señalaron a Santa Bárbara, Fandango y Bahía a las que se aplicaron criterios más específicos Pumalín como las tres opciones más favorables,

[3] Observatorio de Ciudades UC, «Consultoría para el Desarrollo de Lineamientos Estratégicos de Reconstrucción / Relocalización y Plan Maestro conceptual Posdesastre Chaitén», Santiago 2009. Disponible en http://www. regiondeloslagos.cl/noticias/documentos chaiten.php 
3. Ubicación de las localidades de Chaitén y Santa Bárbara dentro del esquema conectividad Provincia de Palena.

4. Foto aérea donde se aprecia el volcán Chaitén, la localidad de Chaitén, en la desembocadura del río Blanco al surponiente del volcán, y la localidad de Santa Bárbara hacia el norponiente. Fuente: fotografía NASA.

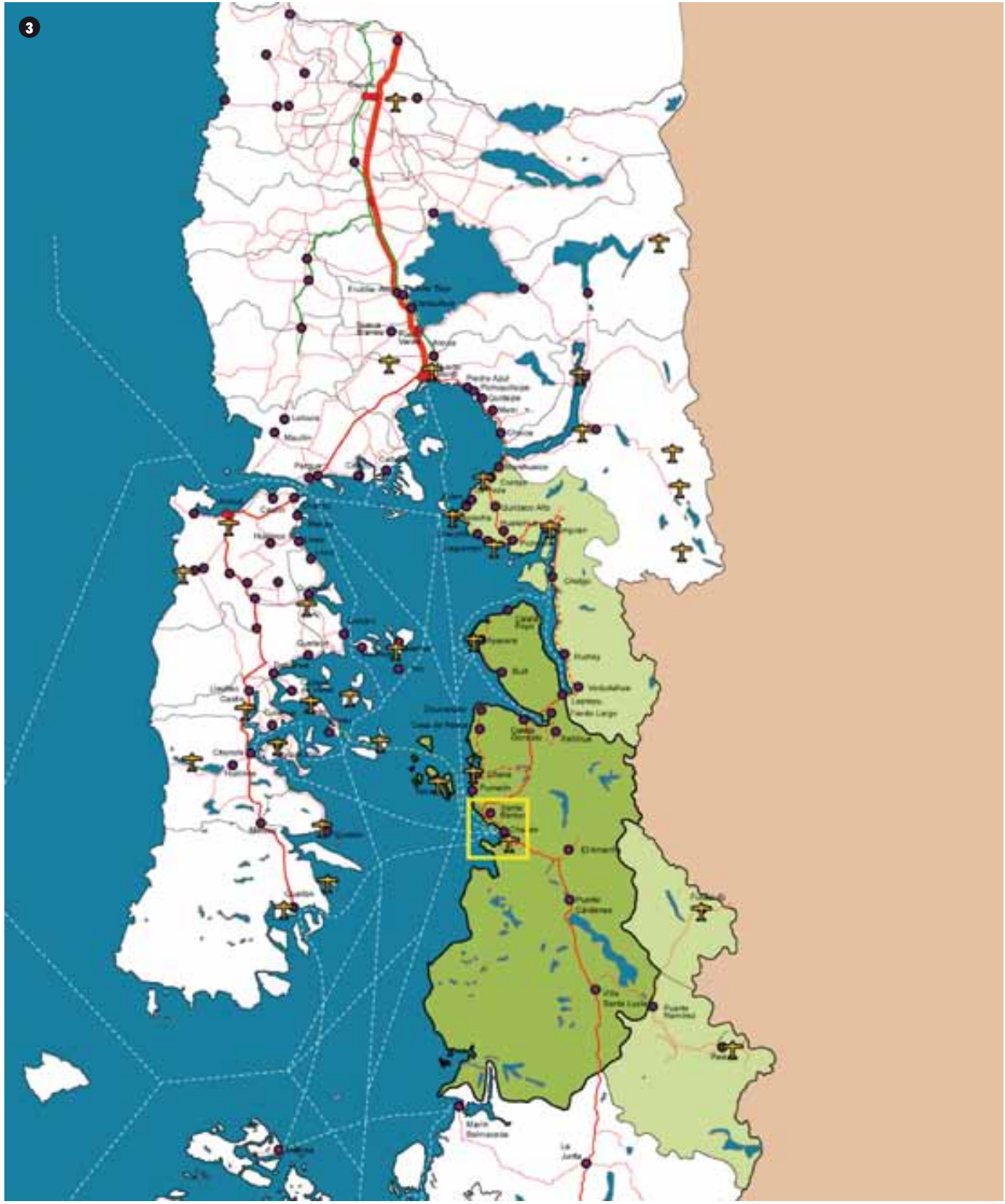

de evaluación incluyendo un análisis de cabida de las demandas de suelo de la ciudad.

Aunque desde el punto de vista de los criterios de menor riesgo y atributos ambientales y paisajísticos las tres localidades presentaron buenos indicadores. La conectividad interprovincial de Santa Bárbara y Fandango tiene claras ventajas sobre la de Pumalín (por tener una ubicación que minimiza tiempos y costos de transporte promedio en la provincia), lo que hizo descartar esta última en favor de las dos primeras. El estudio entregado el año 2009 definió como mayor potencial de desarrollo a Santa Bárbara.

Esta localidad no tuvo indicadores con bajo los temas que estructuran los criterios analizados (medio ambiente, economía y viabilidad, cultura potencial. Esta apreciación es consistente para

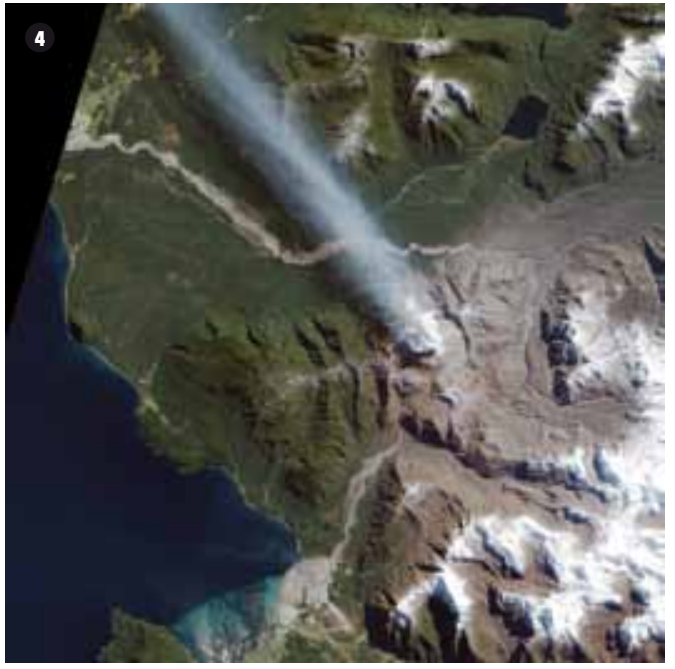

y sociedad y recursos naturales). La mayor cantidad de criterios con impacto positivo están en el área de medio ambiente, como por ejemplo el asoleamiento (Santa Bárbara presenta mayor asoleamiento que Chaitén, por tanto más horas de luz diaria).

La conectividad interprovincial de Santa Bárbara, como se ha mencionado, es muy favorable y tiene ligera ventaja por sobre Fandango por estar más cerca del potencial futuro aeródromo, en desarrollo por el Mop.

\section{UN PROYECTO DEL ESTADO}

Una vez entregado el estudio, la Presidenta Bachelet encarga al Ministerio de Vivienda y Urbanismo el desarrollo de un Plan Maestro para la nueva ciudad, a través de su División de Desarrollo Urbano, en forma conjunta con el Departamento de Desarrollo Urbano e Infraestructura de la SeREMI de Vivienda y Urbanismo de la Región de Los Lagos, el Serviu de la Región de Los Lagos, la Oficina de Desplazados de Chaitén y la Intendencia de la Región de Los Lagos.

El Plan Maestro pasó a ser una excepción dentro de la dinámica del Ministerio de Vivienda y Urbanismo de la época, que poco tiempo antes había diluido el Departamento de Proyectos Urbanos (DPu) aduciendo a la mayor eficiencia del mundo privado para la ejecución de los proyectos. Faltando menos de nueve meses para el término del gobierno, y considerando 
la urgencia de dar respuesta a la carencia provincial de Palena, se decidió hacerlo de manera interna.

Se estableció un equipo dirigido por el Jefe de la División de Desarrollo Urbano, Luis Eduardo Brescianni L., con un equipo de arquitectos ${ }^{[4]}$, tanto a nivel nacional como regional, complementado con un equipo de especialistas que desarrollaron estudios de riesgos, un estudio de energías y estudio preliminar de ingenierías ${ }^{[5]}$.

El Plan Urbano fue planteado como una «herramienta que guía la toma de decisiones para el desarrollo de la nueva ciudad bajo la misión de recuperar la capital de la Provincia de Palena desarrollando una ciudad con una fuerte identidad paisajística y cultural, que incorpore criterios de sustentabilidad ambiental, social y económica, que garantice una alta calidad de vida para sus habitantes, y que se consolide como la puerta de acceso a la Carretera Austral»»[6].

Si bien la intención de la relocalización de la capital provincial respondió a una necesidad generada por una emergencia, al mismo tiempo se explicitó el desafío de aprovechar la inevitable relocalización como oportunidad de revertir los procesos de segregación territorial de la provincia de Palena, generando las inversiones y programas públicos que permitan elevar las condiciones de vida de miles de habitantes de estas zonas, mediante la construcción de un centro urbano de alto estándar que sirva a todas las localidades de la provincia.

En un inicio, en paralelo a recabar información sobre las condiciones del lugar, se desarrolló un proceso de participación en base a la red de vínculos establecida por la Oficina de Desplazados de Chaitén, la cual coordinaba la atención y la ayuda del Estado a los habitantes de la antigua ciudad. Éste se llevó a cabo
5. Localidad de Santa Bárbara, fotografía de 30 de marzo de 2008. Fuente: Minvu X Región.

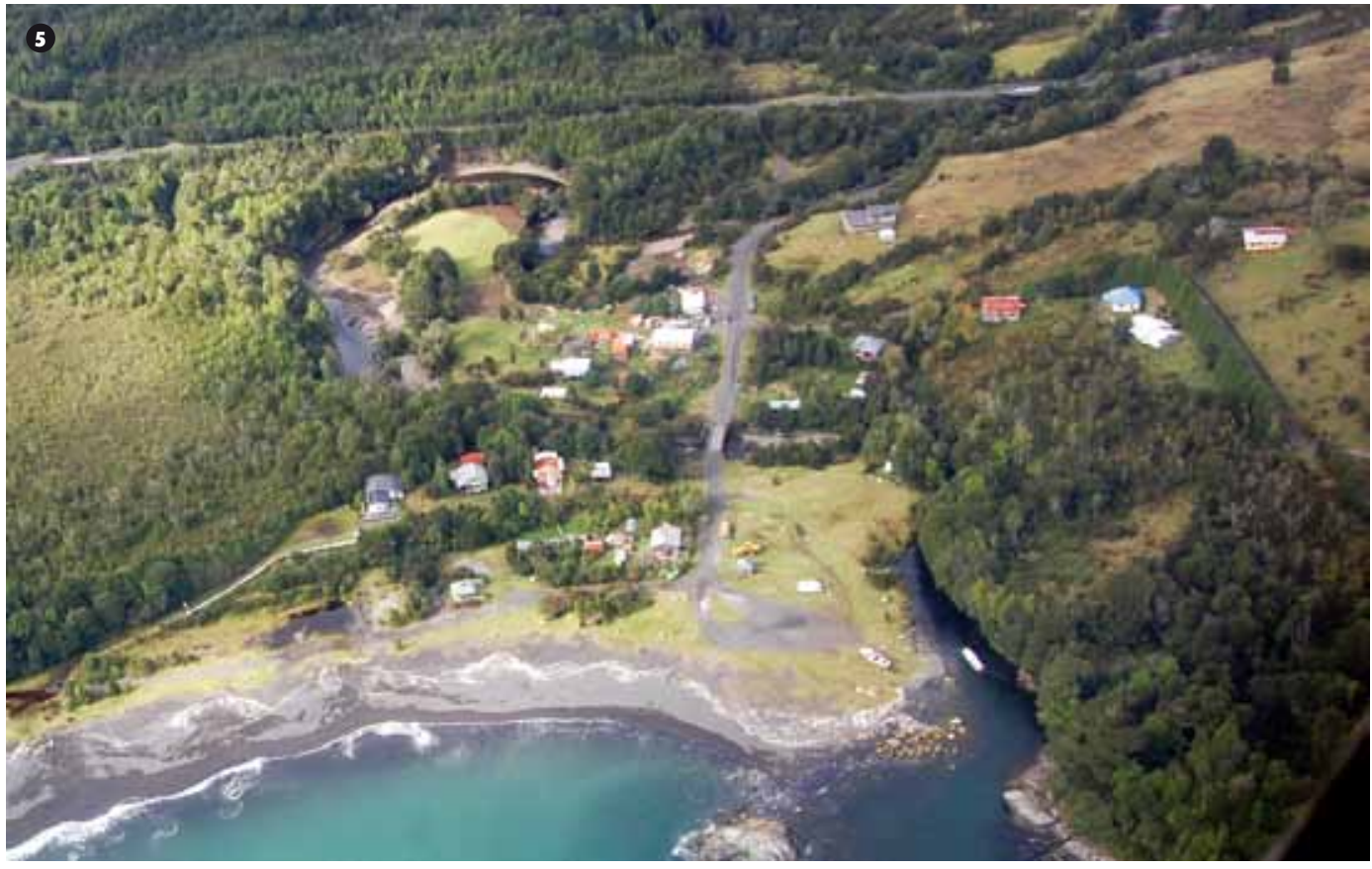

en 14 sesiones realizadas en los lugares con mayor concentración de desplazados ${ }^{[7]}$; si bien no se contaba con ninguna certeza sobre si los que participaban en las sesiones de trabajo regresarían a Chaitén, era necesario registrar de primera fuente una caracterización de los modos de vida de la región.

Así, se buscó que el Gobierno en conjunto con la ciudadanía, por medio de antecedentes existentes y espacios de diálogo y participación, pudieran consensuar opiniones que establecieran las bases de una ciudad que conviva de modo sustentable con su entorno natural y territorial. Para ello se establecieron tres objetivos orientadores de este proceso: reconocer aspectos de identidad de la ciudad de Chaitén, recoger la opinión y expectativas sobre la nueva ciudad de la comunidad desplazada y afectada y establecer acuerdos sobre el carácter y organización de la nueva ciudad ${ }^{[8]}$.

\section{LA ERRADICACIÓN DEL ESTADO}

El gobierno de Sebastián Piñera, asume con la nueva emergencia del 27 de febrero de 2010: un terremoto que ve afectadas a cinco regiones. En altos cargos del Ministerio de Vivienda y Urbanismo se designan a dos actores participantes del estudio de relocalización de la nueva ciudad de Chaitén. Como Subsecretario nombran a Andrés lacobelli, quien consultó para el estudio todo lo referido a transportes, y como asesor principal en temas asociados a la Reconstrucción a Pablo Allard, quien dirigió el Estudio de Relocalización.

Se confirma al Ministerio de Vivienda y Urbanismo como la institución encargada de coordinar y llevar a cabo el proyecto en Santa Bárbara, por medio de oficio por el Subsecretario de Desarrollo Regional ${ }^{[9]}$. El proyecto entra en su fase de implementación

\footnotetext{
Equipo del cual fui parte.

Ver agradecimientos en MINvU, Plan Maestro Ciudad Chaitén, Santiago-Puerto Montt, marzo de 2010.

Minvu, Plan Maestro Ciudad Chaitén, Santiago-Puerto Montt, marzo de 2011

Valdivia, Osorno, Alerce, Puerto Montt, Ancud, Castro, Chonchi, Dalcahue, Achao, Quellón, Futaleufú, Palena, Villa Santa Lucía y La Junta.

Oficina de Apoyo a Desplazados de Erupción Volcán Chaitén, «Proceso de Participación Ciudadana», en Minvu, Anexos Plan Maestro Ciudad Chaitén, marzo de 2011.

9] Oficio Min. Interior n ${ }^{\circ} 1.716$ del 10 de junio de 2010.
} 
6. Plano Imagen Objetivo, Plan Maestro Santa Bárbara. Fuente: Minvu, Plan Maestro Ciudad Chaitén, Informe final, marzo de 2010

7. Vista vuelo de pájaro, Plan Maestro Santa Bárbara. Fuente: Minvu, Plan Maestro Ciudad Chaitén, Informe final, marzo de 2010

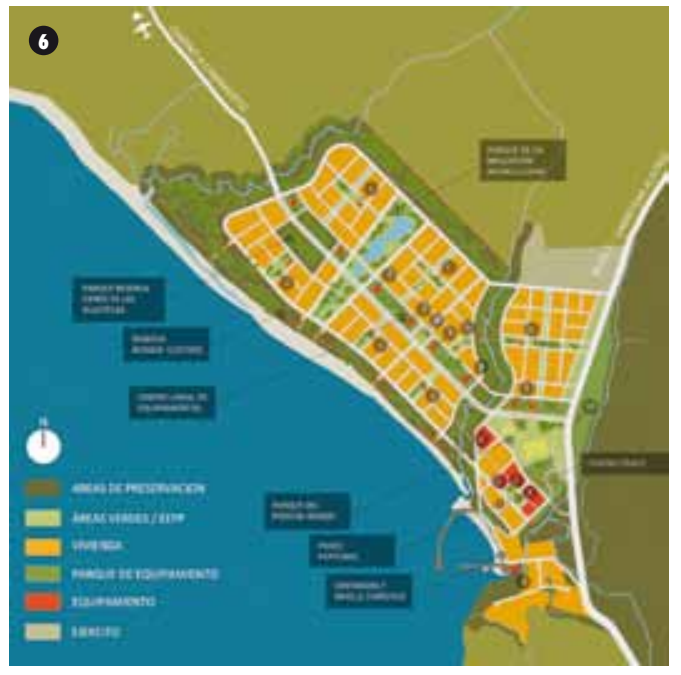

a partir de la coordinación de los distintos actores participantes, tanto a nivel nacional como regional, involucrando a los Ministerios de Bienes Nacionales, al de Obras Públicas, el Municipio de Chaitén, el Ejército, Salud, Educación, Carabineros y a la sociedad civil, por medio de la constitución de un Comité de Vivienda de 140 familias dispuestas a asentarse en Santa Bárbara, aparte de las 300 familias de los funcionarios públicos.

En diciembre de 2010 estaba todo dispuesto para iniciar los estudios de ingeniería para la construcción del macroloteo, habiendo sido evaluados económica y socialmente por MIDEPLAN y aprobados para empezar a ser ejecutados, superando un proceso largo y complejo (cualquiera que conoce el Sistema Nacional de Inversiones entiende del proceso) y con dinero en los presupuestos de los distintos ministerios.

Con el inicio del gobierno de Sebastián Piñera también es nombrado un nuevo intendente en la Región de Los Lagos, Juan Sebastián Montes. La primera acción del Intendente fue juntarse con los habitantes de Chaitén que persistieron en quedarse, rechazando el plan de compensación planteado tras la emergencia. Éste incluía bonos, subsidios y la Ley Chaitén que permitía al Estado, por medio del Ministerio de Bienes Nacionales, la compra de los bienes inmuebles de la localidad. Los habitantes reclamaron la falta de apoyo del Estado al negar el acceso a los servicios básicos (agua y electricidad). Luego de esta reunión y en el contexto de la nueva catástrofe del 27 de febrero, el Intendente

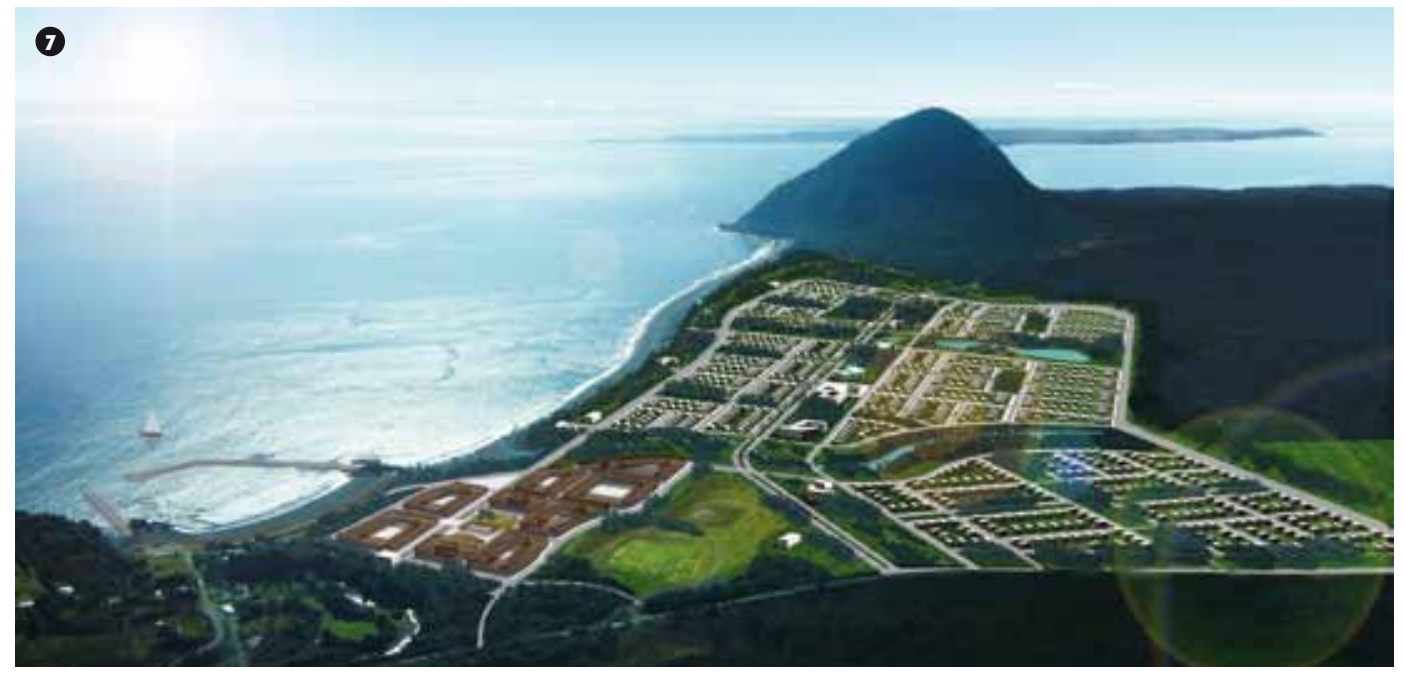

anunció que descartaría el proyecto de Santa Bárbara $^{[10]}$.

Se plantearon razones presupuestarias para suspender el proyecto, como el alto costo del proyecto heredado del gobierno anterior (US\$300 millones), argumentando que ahora no sería así y que se gastarían (US $\$ 40$ millones), lo que siempre correspondió a la misma primera etapa del proyecto del Minvu. A mediados de diciembre de 2010 el Intendente de la Región de Los Lagos anuncia por medio de medios de comunicación regional ${ }^{[1]]}$ el triunfo de «David contra Goliath»: el triunfo del pueblo contra el estado perverso que fracasa en imponer un modo de vida por medio de una ciudad que nadie pidió.

Estos argumentos, con tonos de reivindicación personal, fueron planteados en el corolario de la última decisión presidencial de desechar el proyecto de Santa Bárbara de Chaitén, transmitida por la misma autoridad regional, 6 meses después que el presidente Piñera prometiera los primeros edificios construidos en Santa Bárbara para el año $2012^{[12]}$ :
Desde mi apreciación, la decisión tomada:

a. No consideró los informes de riesgo del SERNEAGEOMIN que ponen a la totalidad de la localidad de Chaitén en zona de riesgo máximo, lo que traería como consecuencias que ni un solo banco tendría garantías para entregar un crédito de inversión para el lugar.

b. Se desestimó el estudio de relocalización firmado por el actual Subsecretario del Minvu, y por el coordinador de la misma cartera para la reconstrucción.

c. Se desestimaron todos los trabajos acordados y coordinados de un sinnúmero de entidades del gobierno tanto a nivel regional como nacional (MINVU, SUBDERE, Bienes Nacionales, Mop, Ejército, Conaf).

d. Se convocó un concurso de «lmagen Urbana» con inversión sobre los $\$ 20$ millones para ser desechado antes de su premiación.
[10] Diario El Mercurio, 27 de mayo de 2010, «Gobierno desechó proyecto de relocalización de Chaitén en Santa Bárbara por alto costo», en http://diario.elmercurio.cl/2010/05/27/nacional/_portada/noticias/f98f3393-c82a4a37-b052-066993d42ceb.htm

[11] SurLink, 13 de diciembre de 2010, http://www.surlink.cl/index.php? option=com_content\&view=article\&id=7053:co mienza-un-nuevo-futuro-para-chaiten\&catid $=42$ :tribuna\&ltemid $=59$

[12] Radio Biobío, http://www.radiobiobio.cl/2010/05/27/sebastian-pinera-confirma-decision-de-emplazar-nuevachaiten-en-santa-barbara/ 
8. Fotomontaje Parque del pozo de áridos, Plan Maestro Santa Bárbara. Fuente: Minvu, Plan Maestro Ciudad Chaitén, Informe final, marzo de 2010.

9. Fotomontaje Plaza Cívica, Plan Maestro Santa Bárbara. Fuente: MINvU, Plan Maestro Ciudad Chaitén, Informe final, marzo de 2010.

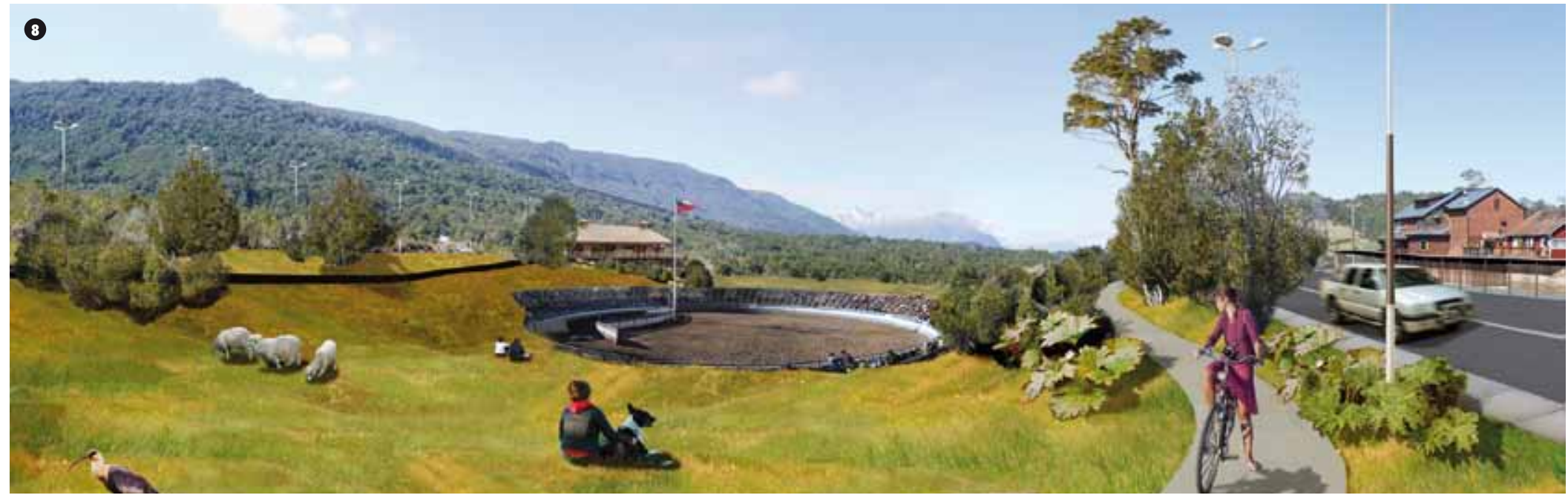

\section{UNA CAPITAL PARA UN TERRITORIO REMOTO}

Palena está lejos y esa es su virtud en un mundo que se achica y se desbasta.

Es un territorio crudo y hostil de topografía extremadamente difícil para los asentamientos humanos, con un clima adverso de lluvias interminables como bien se percató el empresario ecologista Douglas Tompkins, quien vio en este lugar la oportunidad de llevar a cabo su ideal ecológico de protección de un entorno en estado virgen con la mínima presencia de personas, adquiriendo aproximadamente 200.000 hectáreas en la provincia.

Su principal centro poblado era Chaitén el cual se caracterizaba por contar con un porcentaje amplio de la población (en torno al 22\%) dedicado a ser empleado u obrero del sector público, ya sea dependiente del Gobierno Central o de la Municipalidad.

El desarrollo turístico sustentable y su intrínseca protección y valorización de sus ecosistemas están arraigados en los habitantes de esas tierras y es el motor económico existente. La oportunidad de tener una ciudad sustentable y segura, con proyección a largo plazo y compatible con su delicada naturaleza, y propia de un país que quiere hacer las cosas bien, se tiene en las manos y se desecha.

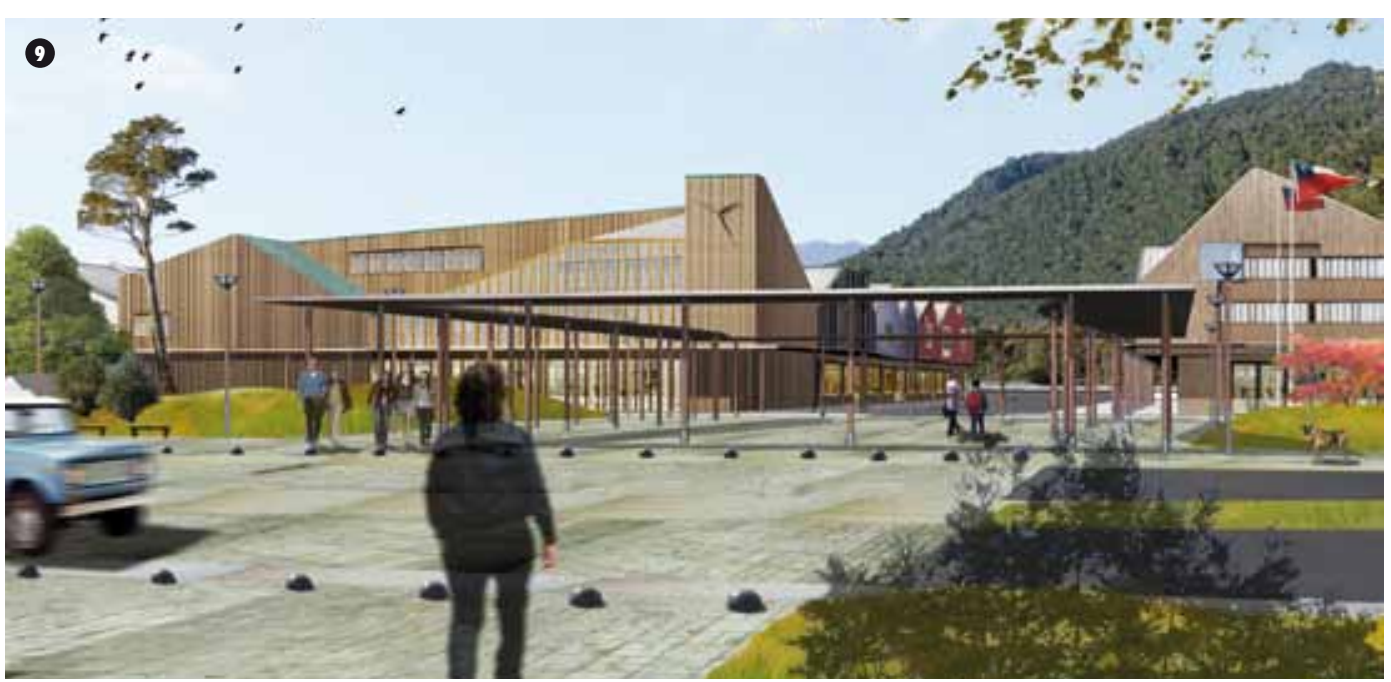

El problema que se arguyó para la toma de decisión, de si se deja libertad o no a las personas que persistieron en quedarse de Chaitén permitiendo y pagando a las empresas de servicios que vuelvan a dotar de agua y electricidad, es cuestionable, desescalado y con gran parte de las aristas sin solución. El problema es, si es necesario invertir en Palena de modo de propiciar un desarrollo integral de una provincia del tamaño de Chiloé con una visión multiescalar y a largo plazo, facilitando las herramientas para su desarrollo compatible a su lema «La Provincia de los Parques».
Es una misión del Estado velar más allá de los problemas individuales de los ciudadanos, pensando tanto en el territorio local, el nacional, y hoy más que nunca, el global; pensando tanto en el ahora, como en cincuenta años más.

Los «Goliaths ganándole a Davides» son fantasmas inventados. Terminadas las emergencias, y con el tiempo y experiencia pasada como patrimonio, es hora de liderar los procesos complejos que implican el desarrollo de un territorio, incluyendo tanto sus habitantes como sus ecosistemas. Así un día lo planteó un sacerdote capuchino venido de Suiza, en una región remota, del extremo sur del mundo. 